\title{
Test of X-band AutoSAR Using Measurement
}

\section{Instruments}

\author{
Hyeon-Cheol Lee, Sang Gyu Lee and Seung Hoon Lee \\ Payload Electronics Team, Satellite Payload Research Office, Satellite R\&D Head Office, Korea Aerospace Research Institute, \\ 169-84, Gwahak-ro, Yuseong-gu, Daejeon, 34133, Rep. of Korea
}

\begin{abstract}
We introduce the stripmap mode test of X-band AutoSAR (automobile synthetic aperture radar) prototype in this paper. We design and develop a power amplifier, a signal demodulator, and use Matlab for post-processing. The transmitter of this prototype, however composes measurement instruments, an Arbitrary Waveform Generator (N8241A, Keysight) for chirp generation and a Vector Signal Generator (E8267D, Keysight) for modulation. Moreover, the COTS (Commercial-Off-The-Shelf) is used for data recording in this test. The resolutions of the prototype finally are $0.41 \mathrm{~m}$ range, $0.14 \mathrm{~m}$ azimuth.
\end{abstract}

Key words: SAR, radar, RailSAR, AutoSAR, stripmap mode, spotlight mode.

\section{Introduction}

There are many devices to observe subjects on the earth from a satellite, such as an optical camera, an infrared camera, an SAR (synthetic aperture radar) [1, 2], etc. The optical camera can find targets clearly, but it has limitation when it is rainy, cloudy, snowy, and dark. The infrared camera can observe targets in the night though, it also cannot distinguish objects when it is rainy, cloudy, and snowy. The SAR, however can observe and monitor the targets regardless of rain, cloud, snow, and darkness. In addition, it can detect underground objects at certain depth, such as tunnels. But, the weak point of SAR image is that the target image is not clearer than the optical camera image.

The SAR payload of KOrea Multi-Purpose SATellite (KOMPSAT)-5 made by Alenia, Italy was launched and is working successfully and then, the development of SAR payload of KOMPSAT-6 is driven by Korean industry so that the interest and demands of SAR developments are increasing now in Korea.

Pieraccini et al. [3] suggested ground based radar

Corresponding author: Hyeon-Cheol Lee, Ph. D., principal researcher, research fields: communications, SAR. techniques. Pieraccini et al. [4, 5] and Ji et al. [6] also suggested the GB-SAR (Ground-based SAR) using a RailSAR for topographic mapping application. Nico et al. [7] and Leva et al. [8] suggested the interferometry test with the GB-SAR systems. Cho et al. [9] had designed and developed the GB-SAR mounted on an automobile. In this paper, we construct the AutoSAR (Automobile Synthetic Aperture Radar) prototype with measurement instruments and perform the stripmap mode [1, 2, 10, 11] test. This AutoSAR provides wide observation area, whereas the RailSAR of which observation area is narrow.

The SAR equipment consists of chirp generator, signal modulator, power amplifier, antenna, signal demodulator, signal processor, and post-processing by software for image. In this test, we use measurement instruments of the Keysight (The previous name was Agilent.) for the functions of chirp generation and modulation. The reason for this was saving monetary budget and manufacturing time of new device.

The SAR image processing of the satellite is not processed onboard, but it transfers by wireless datalink to ground station for post-processing. The amount of SAR raw data is large so that compression techniques are studied much for a long time due to 
data limitation of datalink. But we do not consider this technique here.

This paper organizes as follows: Section 2 introduces the combination of SAR equipment and test scheme, Section 3 shows the test concept of AutoSAR. Section 4 describes test results and analysis. Section 5 concludes.

\section{Description of Test Scheme}

The equipment (see Fig. 1) of SAR consists of a chirp generator that produces linear FM chirp pulses, a signal modulator that converts its signal to center frequency, a power amplifier to amplify the magnitude of the signal, an antenna to emit its energy, a signal demodulator that demodulates and detects the reflected signals, a signal processor that stores in order and processes properly to be sent to the ground. In addition, the post-processing software manages SAR image with the given data.

For the chirp generation and the modulation, we use measurement instruments, an Arbitrary Waveform Generator (AWG N8241A, Keysight) that has an option of linear chirp pulse generation and a Vector Signal Generator (VSG E8267D, Keysight) to convert its baseband signal to X-band. Using AWG and VSG has given us some advantages, such as a stable trigger and changing a bandwidth, a carrier frequency, and a
PRF easily, etc.

We make use of COTS, “Andale”(high speed customized 24TByte) with X6-GSPS FPGA for its data recording and signal rearrangement.

Finally, we employ range compression, range cell migration compensation, and azimuth compression from the Range Doppler Algorithm [1, 2] by Matlab for post-processing to produce SAR images.

Hence, we design and develop a power amplifier and an X-band signal demodulator, subsequently interface them with each equipment. We apply IF sampling and DDC (Digital Down Conversion) scheme here, the sampling rate is over gigahertz. System parameters are summarized in Table 1.

\section{AutoSAR Test}

For the SAR processing, we need the Doppler effect so that we install every equipment inside van like Fig. 2. We mount antenna on the van, separate transmitting and receiving antenna, that means we do not use a circulator so that this scheme reduces RF losses. Although, SAR is usually operated from an aircraft or a satellite for Doppler effect, moving antenna by a car along the horizontal direction is proposed here. This test plot calls the AutoSAR. The test result of the RailSAR which has the point interval emission on the rail is omitted in this paper.

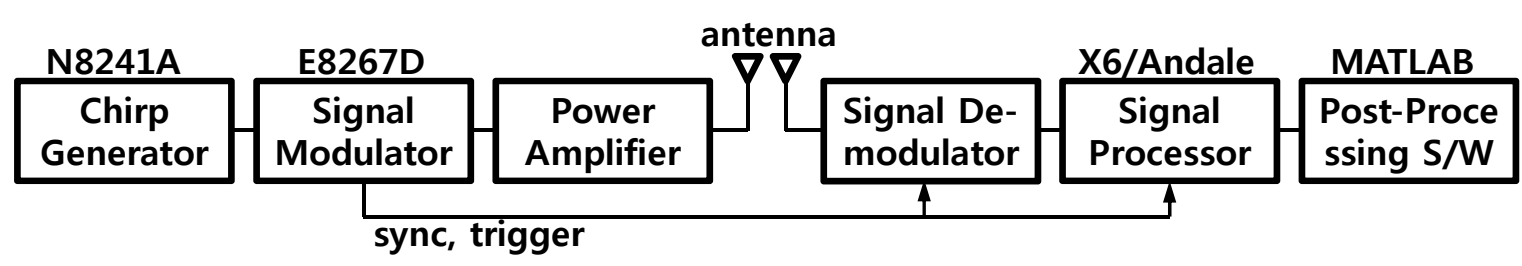

Fig. 1 Block diagram of AutoSAR prototype.

Table 1 Parameters of AutoSAR prototype.

\begin{tabular}{|l|l|}
\hline Parameters & Values \\
\hline Center Freq. & X-band \\
\hline Power Amp. & - \\
\hline Max. Range & $4 \sim 5 \mathrm{~km}$ \\
\hline Sensing Speed & $80 \mathrm{~km} / \mathrm{h}$ av. \\
\hline Antenna Gain & $25 \mathrm{~dB}$, two horn antenna used \\
\hline Beam width & $20 \mathrm{deg}$ \\
\hline
\end{tabular}




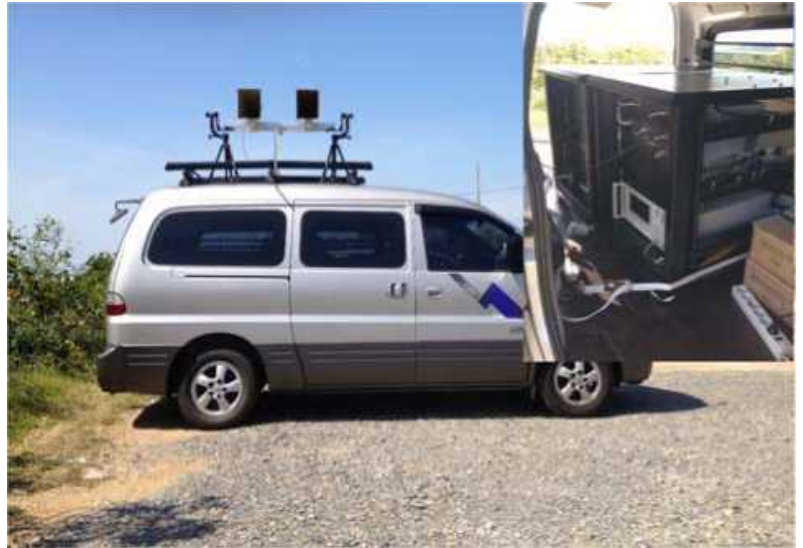

Fig. 2 Configuration of the AutoSAR test.

\section{Results and Analysis}

Several SAR images are obtained. Fig. 3 shows the test results from the seawall, the vertical is an azimuth direction, the horizontal is a range direction that is the direction of the AutoSAR movement.

Fig. 3 is the AutoSAR test results (speed: $80 \mathrm{~km} / \mathrm{h}$ ) on the seawall of which the height is $5 \mathrm{~m}$ high. In Fig. 3 , the upper one shows a VV (Vertical-Vertical) mode image, the middle one shows a $\mathrm{HH}$ (HorizontalHorizontal) mode image, and the last one is an optical camera map. By changing mounting angle of antenna, we get different polarization modes. The VV mode image shows streams of waves by wind, on the contrary, the HH mode image does not, moreover it shows ghosts, even two test conditions are same.

Fig. 4 is a zoom-in image of Fig. 3. The objects in white square line are magnified in the lower figure, the two parallel white lines indicate $1 \mathrm{~m}$ gap bridge. The CY and CR are a cylinder and a reflector respectively.

Fig. 5 shows the SAR image which looks like an airborne SAR image, because the AutoSAR movement is from an $180 \mathrm{~m}$ high bridge. The vertical is the azimuth direction which is the movement of AutoSAR. The radar shadowing in the left upper corner of the image is visible due to side-looking SAR. Some autumn harvest areas in SAR image are not on an optical camera map.

Fig. 6 is the SAR image which shows many radar

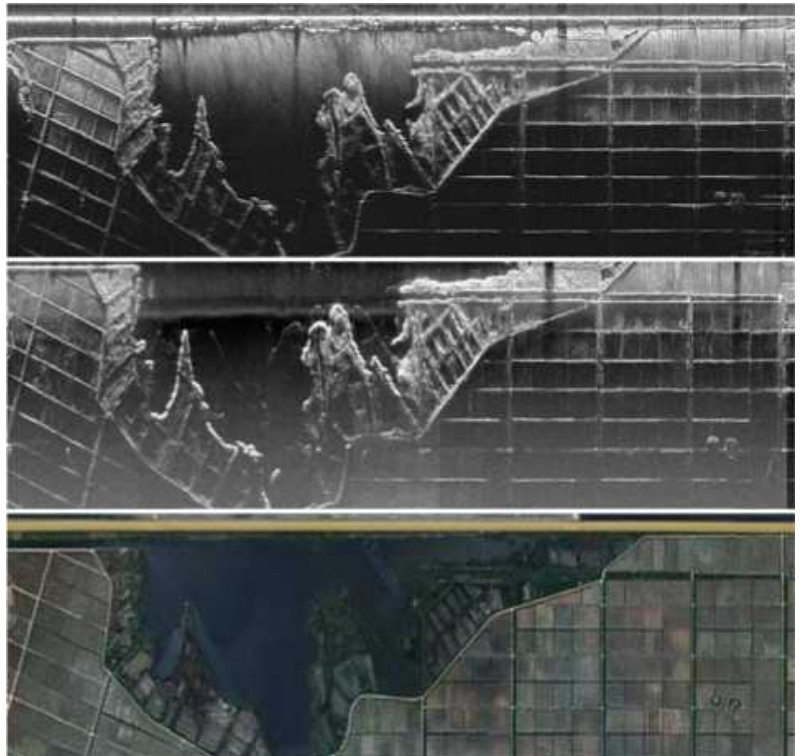

Fig. 3 SAR image from seawall (height $5 \mathrm{~m}$ ), SAR(VV, upper), SAR (HH, middle), Map (below), 4 km × $1.5 \mathrm{~km}$.

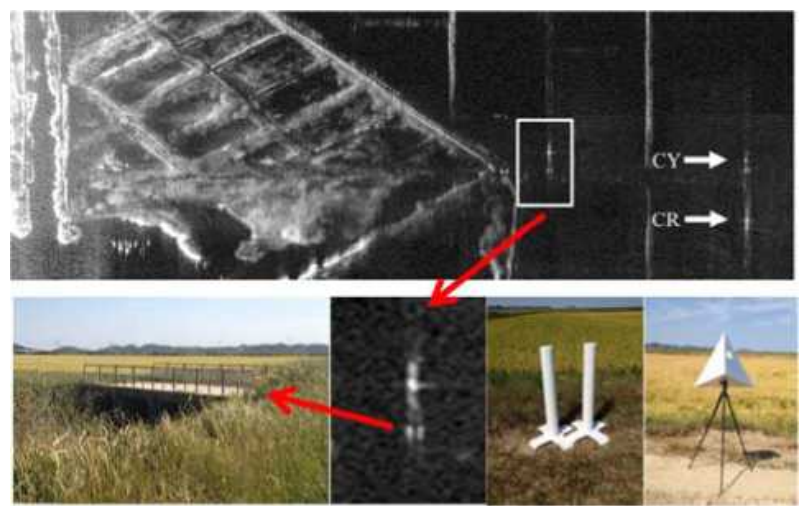

Fig. 4 SAR zoom-in image of Fig. 3.

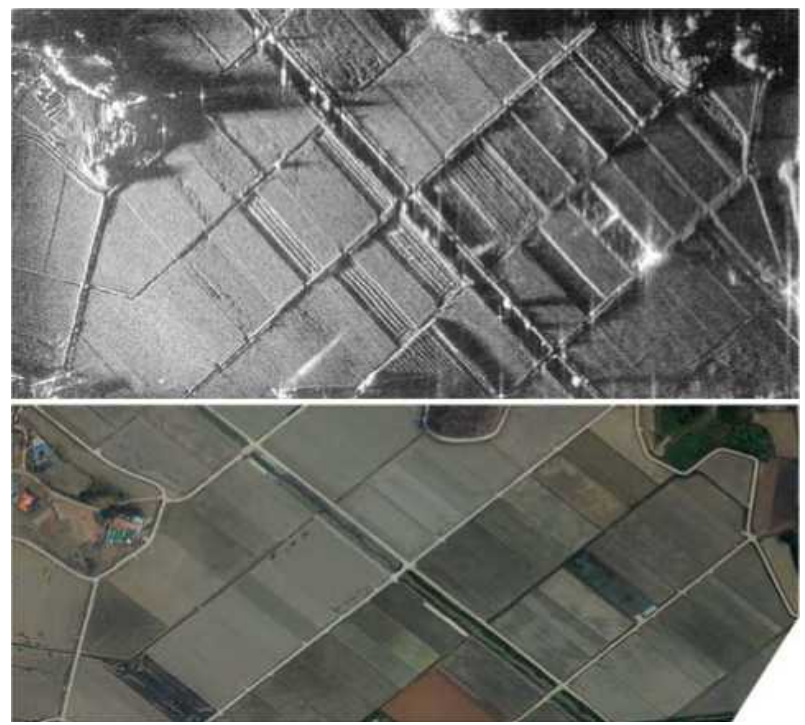

Fig. 5 SAR image from bridge (height $180 \mathrm{~m}$ ). SAR (upper), Map (below). 

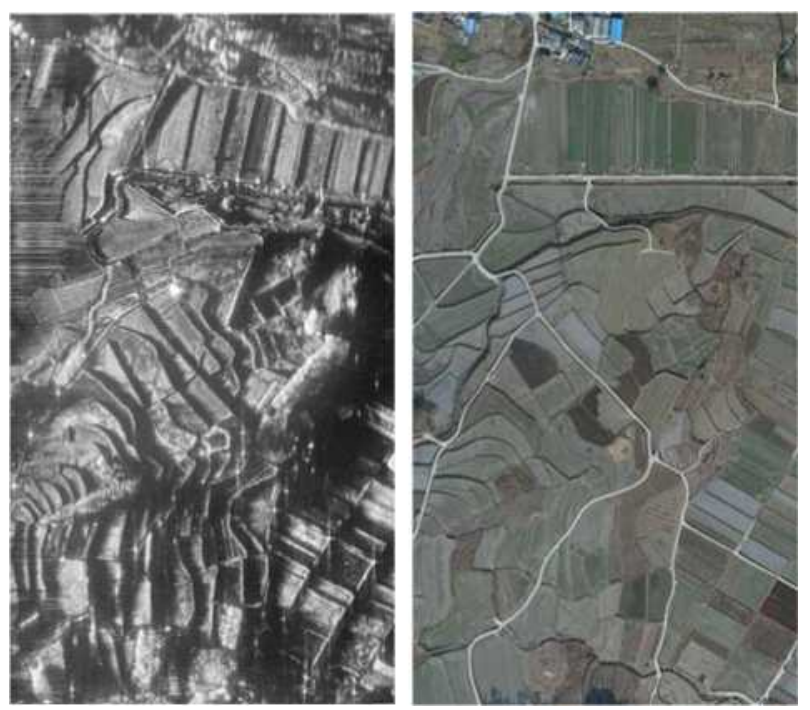

Fig. 6 SAR image from highway (height $20 \mathrm{~m}$ ). SAR (left), Map (right), $0.5 \mathrm{~km} \times 1 \mathrm{~km}$.

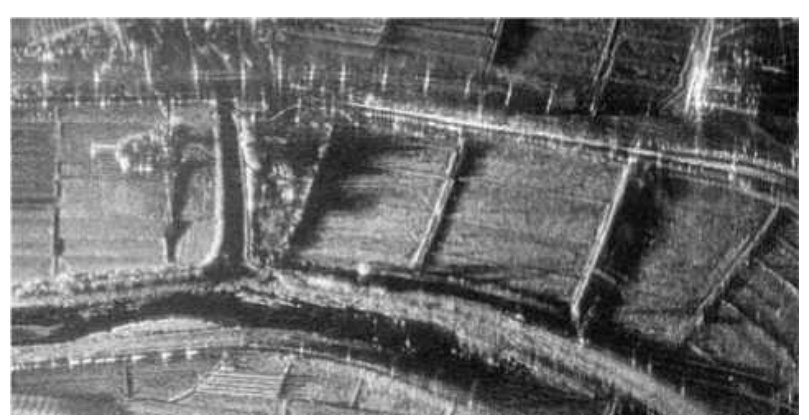

Fig. 7 SAR image (shows railways).

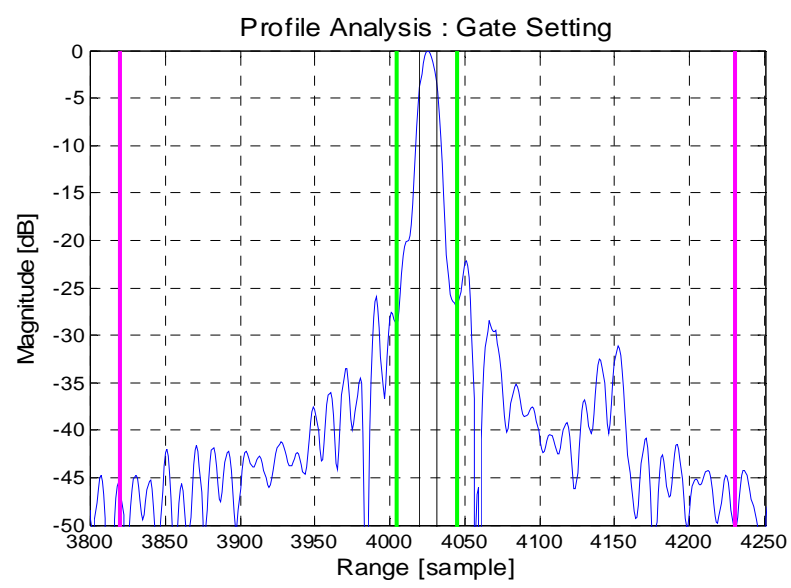

Fig. 8 IRFs in range direction.

shadowings because the AutoSAR movement is from at most $20 \mathrm{~m}$ highway road. This is tested in the night, but the image clearly shows every target, this appeals to the strong point of SAR.

In the upper area of Fig. 7, two fine long lines (railways) are seen, this describes how high the resolution

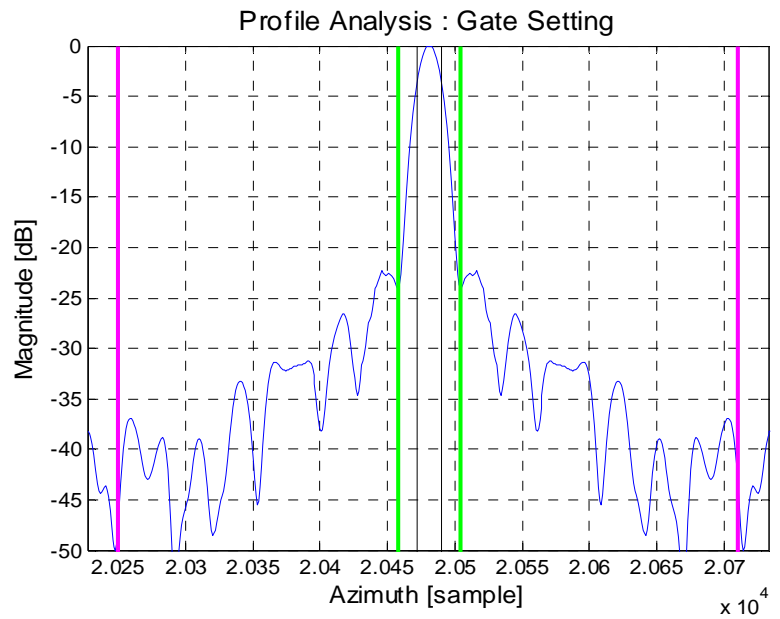

Fig. 9 IRFs in azimuth direction.

Table 2 System resolutions of the AutoSAR test.

\begin{tabular}{lllll}
\hline & \multicolumn{2}{c}{ Range } & \multicolumn{2}{c}{ Azimuth } \\
\cline { 2 - 5 } & Target & Measure & Target & Measure \\
\hline Res & $0.5 \mathrm{~m}$ & $0.41 \mathrm{~m}$ & $0.4 \mathrm{~m}$ & $0.14 \mathrm{~m}$ \\
PSLR & $-12.8 \mathrm{~dB}$ & $-22.1 \mathrm{~dB}$ & $-12.8 \mathrm{~dB}$ & $-22.3 \mathrm{~dB}$ \\
ISLR & $-8.8 \mathrm{~dB}$ & $-20.2 \mathrm{~dB}$ & $-8.8 \mathrm{~dB}$ & $-17.1 \mathrm{~dB}$ \\
\hline
\end{tabular}

of this system is. Several vertical lines on the rails are electric power towers.

We measure the resolution of this system with IRF (Impulse Response Function) (see Figs. 8 and 9)[12], the range resolution is $0.41 \mathrm{~m}$, the azimuth resolution is $0.14 \mathrm{~m}$ in Table 2 .

\section{Conclusions}

We designed and developed an AutoSAR prototype and tried the stripmap mode. We simply composed the $\mathrm{X}$-band AutoSAR prototype with measurement instruments for chirp generation and modulation, and COTS for data recording. We, furthermore used the Range Doppler Algorithm by Matlab for the post-processing of the SAR image. After measuring IRF, we got $0.41 \mathrm{~m}$ range resolution, $0.14 \mathrm{~m}$ azimuth resolution, better than our target resolutions (0.5 $\mathrm{m}$ (range), $\quad 0.4 \mathrm{~m}$ (azimuth)), it is beyondour expectation.

\section{References}

[1] Cumming, I., and Wong, F. 2004. "Digital Processing of Synthetic Aperture Radar Data.” Artech House.

[2] Curlander, J., and McDonough, R. 1991. Synthetic 
Aperture Radar Systems and Signal Processing. New York, NY: John Wiley \& Sons Inc.

[3] Pieraccini, M., Luzi, G., Mecatti, D., and Atzeni, C. 2003. "A Ground Based Remote Sensing Radar Technique for Dynamic Testing of large Structures.” In Proceedings of the Int.Geosci. and Remote Sens. Symposium(IGARSS) 2003, Toulouse, France, 4326-8.

[4] Pieraccini, M., Luzi, G., and Atzeni, C. 2000. "Ground-Based Interferometric SAR for Terrain Elevation Mapping.” IEEE Electronics Letters 36 (16): 1416-7.

[5] Pieraccini, M., Luzi, G., and Atzeni, C. 2001. "Terrain Mapping by Ground-Based Interferometric Radar.” IEEE Trans. Geosci. Remote Sens. 39 (10): 2176-81.

[6] Ji, Y., Han, H., and Lee, H. 2014. "Construction and Application of Tomographic SAR System Based on GB-SAR System.” In Proceedings of the Int.Geosci. and Remote Sens. Symposium(IGARSS) 2014, Quebec, Canada, 1891-4.

[7] Nico, G., Leva, D., Antonello, G., and Tarchi, D. 2004. "Ground-Based SAR Interferometry for Terrain Mapping: Theory and Sensitivity Analysis.” IEEE Trans. Geosci.
Remote Sens. 42 (6): 1344-50.

[8] Leva, D., Nico, G., Tarchi, D., Fortuny-Guasch, J., and Sieber, A. 2003. "Temporal Analysis of a Landslide by Means of a Ground-Based SAR Interferometer.” IEEE Trans. Geosci. Remote Sens. 41 (4): 745-52.

[9] Cho, B-L., Kong, Y-K., Park, H-G., and Kim, Y-S. 2006. "Automobile-Based SAR/InSAR System for Ground Experiments.” IEEE Geosci. Remote Sens. Letters 3 (3): 401-5.

[10] Spudis, P., Nozette, S., Sussey, B., Raney, K., Winter, H., Lichtenberg, C., Marinelli, W., Crusan, J., and Gates, M. 2009. "Mini-SAR: An Imaging Radar Experiment for the Chandrayaan-1 Mission to the Moon.” Current Sci. 96 (4): 533-9.

[11] Agrawal, N., and Venugopalan, K. 2010. "Saturation Adaptive Quantizer Design for Synthetic Aperture Radar Data Compression.” Int. J. Comp. Sci. Eng. 02 (01S): 76-81.

[12] Lacomme, P., Hardange, J., Marchais, J., and Normant, E. 2001. Air and Spaceborne Radar Systems: An Introduction. Norwich, NY: William Andrew Publishing.. 